\title{
A novel CX3CR1 inhibitor AZD8797 facilitates early recovery of rat acute spinal cord injury by inhibiting inflammation and apoptosis
}

\author{
GUOZHAO CHEN $^{1 *}$, ZHIPING ZHOU ${ }^{1 *}$, WEIPING SHA ${ }^{1}$, LIMING WANG ${ }^{1}$, FEI YAN ${ }^{1}$, XIAOMEI YANG ${ }^{2}$, \\ XIA QIN ${ }^{3}$, MUYAO WU ${ }^{4}$, DI LI ${ }^{5}$, SHOUJIN TIAN ${ }^{1}$ and GANG CHEN ${ }^{6}$ \\ Departments of ${ }^{1}$ Orthopedics, ${ }^{2}$ Emergency and ${ }^{3}$ ICU, The Affiliated Zhangjiagang Hospital of Soochow University; \\ ${ }^{4}$ Department of Rehabilitation, Zhangjiagang Hospital of Traditional Chinese Medicine Affiliated to \\ Nanjing University of Chinese Medicine; ${ }^{5}$ Department of Neurosurgery and Translational Medicine Center, \\ The Affiliated Zhangjiagang Hospital of Soochow University, Suzhou, Jiangsu 215600; \\ ${ }^{6}$ Department of Neurosurgery and Brain and Nerve Research Laboratory, \\ The First Affiliated Hospital ofSoochow University, Suzhou, Jiangsu 215031, P.R. China
}

Received June 21, 2019; Accepted January 31, 2020

DOI: $10.3892 /$ ijmm.2020.4509

\begin{abstract}
The present study aimed to evaluate the effect of the CX3CR1 inhibitor AZD8797 in early recovery after acute SCI and elucidate its potential mechanism in blocking inflammation and apoptosis. Adult rats were sacrificed after 3, 7, 10, or 14 days of SCI. The injured spinal tissues were collected for assessing C-X3-C motif chemokine ligand 1(CX3CL1)/C-X3-C motif chemokine receptor 1 (CX3CR1) expression at each time point via western blotting (WB) and quantitative PCR. The cellular localization of the proteins was detected by immunofluorescence. Another batch of rats (subdivided into sham, injury model, AZD8797 and methylprednisolone groups) were used to evaluate locomotive recovery with a Basso Beattie Bresnahan score. Based on the expression level of CX3CR1, these rats were sacrificed at the most prominent stage of CX3CR1 expression (10 days after SCI), for assessing the serum levels of tumor necrosis factor- $\alpha /$ interleukin (IL)-6/IL-1 $\beta$ and
\end{abstract}

Correspondence to: Dr Shoujin Tian, Department of Orthopedics, The Affiliated Zhangjiagang Hospital of Soochow University, 68 Jiyang West Road, Zhangjiagang, Suzhou, Jiangsu 215600, P.R. China

E-mail: zjgchenguozhao@163.com

Dr Di Li, Department of Orthopedics, Department of Neurosurgery and Translational Medicine Center, The Affiliated Zhangjiagang Hospital of Soochow University, 68 Jiyang West Road, Zhangjiagang, Suzhou, Jiangsu 215600, P.R. China

E-mail: lidilab@sina.com

${ }^{*}$ Contributed equally

Key words: C-X3-C motif chemokine receptor 1, AZD8797, tumor necrosis factor- $\alpha /$ interleukin-6/interleukin-1 $\beta$ signaling, necrosis, apoptosis, spinal cord injury the expression of CX3CL1/CX3CR1/caspase 3/Bcl-2/Bax in the spinal cord tissues through WB and ELISA. Additionally, apoptosis and necrosis in the injured spinal cord were evaluated by terminal deoxynucleotidyl transferase-mediated dUTP nick-end labeling staining/fluoro-jade B staining. Expression levels of both CX3CR1 and CX3CL1 reached their peak 10 days after the injury, followed by a dramatic downward trend at 14 days. The enhanced expression of CX3CR1 was detected in astrocytes and microglia of the injured spinal cord. AZD8797 improved locomotive recovery after 10 days of SCI and was as effective as methylprednisolone. The effect of AZD8797 was mediated by suppressing apoptosis, necrosis and inflammatory responses, as assessed by WB/ELISA and morphological examinations. The current study has demonstrated that AZD8797 can effectively block overwhelming inflammation, apoptosis and necrosis after SCI and facilitate early recovery of locomotive function.

\section{Introduction}

Spinal cord injury (SCI) is one of the most heterogeneous injuries occurring in the central nervous system for its diverse symptoms and treatment outcomes, because of variability in the external mechanical forces causing the injury. Based on the epidemiological data, the World Health Organization has predicted that SCI is most likely to surpass numerous diseases as the major cause of death and disability worldwide by 2020 (1). Moreover, a statistical study by the National Spinal Cord Injury Statistical Center has indicated that the global incidence of SCI is roughly 23 SCI cases per million annually $(2,3)$. Chronic complications after primary SCI are quite common and severe (4-9), leading to reduced life expectancy and enhanced morbidity. This not only creates a physically and emotionally debilitating condition but also generates a prominent financial burden for individuals, families, and society $(10,11)$. Thus, there is an urgent need in research for developing new therapeutics. 
SCI can cause immediate cellular death, resulting from direct mechanical impact or compression and followed by numerous types of secondary and neurodegenerative processes (12-14). Those secondary processes may cause progressive and fundamental alterations of the cellular structure and function. These effects are most likely to occur on the injured sites, where the cells are extremely susceptible to free radical overproduction and lipid peroxidation as well as glutamate-calcium $\left(\mathrm{Ca}^{2+}\right)$ - and potassium $\left(\mathrm{K}^{+}\right)$-related neurotoxicity, followed by a local or systemic inflammatory responses $(12,13,15,16)$. Consequently, all these drastic alterations at the cellular and subcellular level inevitably result in not only accumulative progression of the microglial inflammatory response but also Wallerian degeneration and reactive astrogliosis. Eventually, the fibrotic core surrounded by reactive astrocytes will form glial scars, resulting in progressive and severe loss of motor and sensory function due to structural changes at the neuronal micro-circuitry level (17-19).

Unlike the immediate primary injuries caused by structural deconstruction, secondary injuries, caused by numerous inflammatory mediators, particularly cytokines and chemokines, are progressive, leading to further damage beyond the primary injury. Those chemokines are secreted by resident cells in the central nervous system (CNS) and by infiltrating cells, recruited to the CNS through blood vessels $(20,21)$. As a result of the extensive association of inflammatory mediators with CNS function, chemokines, designated as a novel subtype of neurotransmitters and neuromodulators, have previously received attention under physiological or pathological conditions and, hence, are also known as neurochemokines $(22,23)$. In previous years, several studies independently uncovered that the secondary injury of SCI is closely correlated with immunological components (24). Moreover, the corresponding therapeutic approaches targeting inflammatory responses are quite promising, with enhanced neuroprotection and neuro-regeneration $(25,26)$.

In particular, $\mathrm{C}-\mathrm{X} 3-\mathrm{C}$ motif chemokine ligand 1 (CX3CL1) also known as fractalkine has so far been regarded as the only member of the $\mathrm{CX} 3 \mathrm{C} \delta$ subfamily that has both soluble and membrane-anchored forms. Thus, its dual roles are uniquely fulfilled as both a chemoattractant and a cell adhesion molecule. The latter role is mediated by its binding to the $\mathrm{C}-\mathrm{X} 3-\mathrm{C}$ motif chemokine receptor 1 (CX3CR1), a G protein-coupled receptor (27).

Given its role in mediating communication among neuronal, microglial and astroglial populations, CX3CL1/CX3CR1 signaling plays an important role in hippocampal synaptic plasticity and maturation (28), and this signaling exhibits a remarkable effect on the modulation of human temporal lobe epilepsy (29), glioblastoma $(30,31)$ and CNS injury (32). Previous evidence $(28,32)$ has implicated the role of the CX3CL1/CX3CR1 axis in the pathophysiology of neuroinflammatory processes after CNS injuries such as traumatic brain injury and SCI. The role of the CX3CL1/CX3CR1 axis has not been widely recognized for its presence and potential function in the pathophysiology of SCI-related phenomena, and its importance in systemic and direct local immune responses is still under investigation. The quality of the evidence and the safety of its application has been continually debated. The microglial inflammatory response, in addition to subsequent
Wallerian degeneration and reactive astrogliosis, can dramatically vary following SCI (33-35). Until now, there is little data on how to treat SCI in experimental animals or human patients. A favorable profile of the corresponding treatment via the CX3CL1/CX3CR1 axis is thus far from being considered definitive. Thus, this study focuses on developing a novel therapeutic approach to selectively target the CX3CL1/CX3CR1 axis following SCI and explore the detailed molecular mechanisms for the role of CX3CL1/CX3CR1 in the pathogenesis of secondary injury in SCI.

\section{Materials and methods}

Animal models. Adult male Sprague-Dawley rats $(n=75)$, weighing 220-280 g, were purchased from the Animal Facilities of Soochow University. A total of 65 of them eventually qualified for final statistical analyses. They were kept in cages with controlled temperature $\left(22^{\circ} \mathrm{C}\right)$ and humidity (50-70\%), where food and water were offered ad libitum, along with 12-h light and dark cycle. All experimental procedures were approved and supervised by the Animal Care and Use Committee of the Soochow University, which were practiced in compliance with the guidelines regarding the Care and Use of Laboratory Animals from the National Institutes of Health.

The rat SCI model was generated with a clip compression method as previously described (36). Following anesthesia via intraperitoneal injection with $4 \%$ chloralhydrate $(400 \mathrm{mg} / \mathrm{kg})$, the rat skulls were fixed by a stereotactic instrument. Following a posterior median incision at $\mathrm{T} 10$, the paravertebral muscles were pulled aside from both sides to expose the T9-T11 lamina. The T10 lamina was trimmed and, hence, the bilateral edges of the dura were clearly exposed. After satisfactory hemostasis, a clamp (Lawton; Huanxi) with a closing pressure of $30 \mathrm{~g}$, was applied to directly clamp the spinal cord and then released carefully after $20 \mathrm{sec}$. After surgery, the muscles and the skin were sutured. According to each experimental design as follows, the T10 lamina was resected and the dura mater was exposed. However, no clamp was used in the sham operated rats. For drug treatment, the CX3CR1 inhibitor AZD8797 $(80 \mu \mathrm{g} / \mathrm{kg}$ ) (37) dissolved in DMSO, was injected intraperitoneally after SCI in rats from subset II, once per day until the rats were sacrificed, whereas methylprednisolone was injected intraperitoneally $(30 \mathrm{mg} / \mathrm{kg}$ ) (38) within $30 \mathrm{~min}$ after SCI in rats from the subset II. A bladder massage was given twice a day after the operation until normal urination was restored. The pads were replaced every 2 days after the operation to keep the limbs dry and the limbs with pressure sores were disinfected with an iodophor.

A total of $4 \%$ chloral hydrate $(400 \mathrm{mg} / \mathrm{kg})$ was injected intraperitoneally, blood samples were collected after anaesthesia, then an assistant held the back of the rat, exposing the neck, the rats' head were removed with scissors and the needed spinal cord tissues were collected.

A total of 25 out of 30 surviving rats (subset I), which had no statistical difference concerning their weight, intake and motor ability, were randomly divided into 5 groups, namely sham, SCI 3 day (D), SCI 7D, SCI 10D, and SCI 14D groups (Fig. 1B). The tissues from the spinal lesion area were collected and longitudinally split into two equal aliquots, one of which were used for quantitative(q)PCR and the other for western 
blot analyses. Similarly, 40 out of 45 surviving rats (subset II), which had no statistical difference in weight, intake and motor ability, were randomly divided into 4 groups, namely sham, SCI, SCI + inhibitor (AZD8797), and SCI + methylprednisolone (Me) groups (Fig. 1C). A total of 10 days after SCI, the locomotive recovery was assessed via Basso Beattie Bresnahan (BBB) scoring in all groups before sacrificing. The blood $(\sim 3 \mathrm{ml})$ was then collected from the inferior vena cava after anesthesia in each of those 40 rats. Finally, the rats were sacrificed to collect their spinal cord tissues. In addition, other evaluations, such as terminal deoxynucleotidyl transferase-mediated dUTP nick-end labeling (TUNEL) staining and fluoro-jade B (FJB) staining, were also carried out accordingly (Fig. 1C). All experiments were strictly adhered to blinded methods during the analyses. Meanwhile, all data that was associated with the corresponding samples was recorded by an independent researcher.

qPCR. Using TRIzol reagent (Invitrogen; ThermoFisher Scientific, Inc.), the total RNA was obtained from the spinal cord tissues. The extracted RNA was reverse-transcribed as follows: First, the oligo(dt) primer, mRNA and nuclease-free water were added into a sterile, nuclease-free tube on ice-cold water to make a total volume $12 \mu 1$. The solution was mixed gently, centrifuged briefly $\left(860 \mathrm{x} \mathrm{g} ; 4^{\circ} \mathrm{C} ; 30 \mathrm{sec}\right)$ and incubated at $65^{\circ} \mathrm{C}$ for $5 \mathrm{~min}$. The solution was then chilled on ice, spun down $\left(860 \mathrm{x} \mathrm{g} ; 4^{\circ} \mathrm{C} ; 30 \mathrm{sec}\right)$ and the resulting vial placed back on ice. The 5x Reaction Buffer, RiboLock RNase Inhibitor, 10 mM dNTP Mix, Revert Aid H Minus M-MuLV and Reverse Transcriptase were then added to the solution, and it was mixed gently and then centrifuged $\left(860 \times \mathrm{g} ; 4^{\circ} \mathrm{C} ; 30 \mathrm{sec}\right)$, then incubated for $60 \mathrm{~min}$ at $42^{\circ} \mathrm{C}$. The reaction was terminated by heating the solution at $70^{\circ} \mathrm{C}$ for $5 \mathrm{~min}$. The reverse transcription reaction product was used directly in PCR applications or stored at $-20^{\circ} \mathrm{C}$ for $<1$ week. For longer storage, $-70^{\circ} \mathrm{C}$ is recommended. The Revert Aid H Minus First Strange cDNA Synthesis kit (Thermo Fisher Scientific, Inc.) used for the reverse transcription. cDNA was synthesized from $1 \mu \mathrm{g}$ of the total RNA. qPCR was performed using a QuantStudio ${ }^{\mathrm{TM}}$ Dx Instrument (Life Technologies; ThermoFisher Scientific, Inc.) with a PowerUp ${ }^{\mathrm{TM}}$ SYBR ${ }^{\mathrm{TM}}$ Green Master Mix (Thermo Fisher Scientific, Inc.). The qPCR protocol is as follows: Denaturation of templates was initiated at $95^{\circ} \mathrm{C}$ for $2 \mathrm{~min}$, followed by 40 cycles of the amplification reaction $\left(95^{\circ} \mathrm{C}\right.$ for $15 \mathrm{sec}, 60^{\circ} \mathrm{C}$ for $15 \mathrm{sec}$ and $72^{\circ} \mathrm{C}$ for $1 \mathrm{~min}$ ). GAPDH mRNA was employed as an internal control for each sample tested and relative mRNA expression levels of all genes tested were quantified using the $2^{-\Delta \Delta \mathrm{Cq}}$ method (39) $(n=3)$. In addition, the primers used in the present studywere as follows: CX3CR1: Forward, 5'-GCTGAGGCCTGTTATTTGGG-3'; and reverse, 5'-GAC CGAACGTGAAGACAAGG-3'; CX3CL1: Forward, 5'-TCA TTCAGAAGCTGCCAGGA-3'; and reverse, 5'-AGAGTC CCTTCCAGAACACG-3'; GAPDH: Forward, 5'-TGGCCT TCCGTGTTCCTACC-3'; and reverse, 5'-TCTTCCACCACT TCGTCCGC-3'.

Western blotting. Protein samples were obtained from the spinal cord tissues lysed for homogenization using RIPA buffer (Beyotime Institute of Biotechnology), supplemented with a protease/phosphatase inhibitor cocktail, followed by centrifugation at $13,000 \mathrm{x}$ g at $4^{\circ} \mathrm{C}$ for $20 \mathrm{~min}$. The protein concentration in the supernatants was measured using aPierce $^{\mathrm{TM}}$ bicinchoninic acid Protein Assay kit (Thermo Fisher Scientific, Inc.) and diluted accordingly. Equal amounts of protein $(30 \mu \mathrm{g})$ in all samples were used for electrophoresis with $10 \%$ SDS-polyacrylamide gels (Beyotime Institute of Biotechnology) and then transferred onto polyvinylidene difluoride membranes (EMD Millipore). After blocking with $5 \%$ non-fat milk in $0.1 \%$ TBST for $2 \mathrm{~h}$ at room temperature, the membranes were incubated with the primary antibodies overnight at $4^{\circ} \mathrm{C}$ with the following dilution: Rabbit anti-GAPDH (1:10,000; cat. no. PLA 0125; Sigma-Aldrich; Merck KGaA), mouse anti- $\beta$-actin (1:10,000; cat. no. A5316; Sigma-Aldrich; Merck KGaA), rabbit anti-CX3CL1 (1:1,000; cat. no. ab25088; Abcam), rabbit anti-CX3CR1 (1:1,000; cat. no. ab8021; Abcam), rabbit anti-Bcl2 (1:1,000; cat. no. ab196495; Abcam), rabbit anti-Bax (1:2,000; cat. no. ab232479; Abcam) and rabbit anti-caspase 3 (1:500; cat. no. ab49822; Abcam). The membranes were then incubated with the goat anti-mouse IgG-horseradise peroxidase (HRP) (cat. no. 31430) or anti-rabbit IgG-HRP (cat. no. 31431) (both 1:10,000; both from Invitrogen; Thermo Fisher Scientific, Inc.) secondary antibodies at $4^{\circ} \mathrm{C}$ for $2 \mathrm{~h}$. Immunoblots were then visualized with a chemiluminescent substrate (EMD Millipore) using a Bio-Rad imaging system (Bio-Rad Laboratories, Inc.), followed by image analyses using ImageJ software (version ImageJ 1.44P; National Institute of Health).

Immunofluorescence. The injured spinal cords were dissected out and post-fixed with $4 \%$ paraformaldehyde for $24 \mathrm{~h}$ at $4{ }^{\circ} \mathrm{C}$. The samples were then sequentially dehydrated in 15 and $30 \%$ sucrose in PBS ( $\mathrm{pH} 7.4$ ) for $24 \mathrm{~h}$. Then the samples were embedded in OCT compound (Sakura Finetek USA, Inc.) and frozen at $-80^{\circ} \mathrm{C}$ until use. Coronal sections $(15 \mu \mathrm{m}$ thick) were obtained by cryosectioning with Leica DMi8 (Leica Microsystems, $\mathrm{GmbH}$ ) and transferredonto the slides pre-coated with poly-L-lysine. After rinsing with $1 \%$ Triton in PBS, the sections were incubated in the blocking buffer containing $10 \%$ goat serum at room temperature for $>1 \mathrm{~h}$. Then they were incubated with the primary antibodies overnight at $4^{\circ} \mathrm{C}$ as follows: Mouse anti-cluster of differentiation11b (1:200; cat. no. MABF520; EMD Millipore), mouse anti-glial fibrillary acidic protein (1:300; cat. no. SAB1405864; EMD Millipore), rabbit anti-CX3CR1 (1:100; cat. no. ab8021; Abcam) and mouse anti-NeuN (1:200; cat. no. MAB377; EMD Millipore), followed by incubation with secondary antibodies, including donkey anti-rabbit $\mathrm{IgG}$ antibody conjugated with Alexa Fluor 488 (1:1,000; cat. no. R37118; Invitrogen; Thermo Fisher Scientific, Inc.) or donkey anti-mouse IgG antibody conjugated with Alexa Fluor 555 (1:1,000; cat. no. A-31570; Invitrogen; Thermo Fisher Scientific, Inc.), for $1 \mathrm{~h}$ at room temperature. The results were observed under a Leica DMi8 confocal microscope and captured using LAS X software (version 2.0.1.14392; Leica Microsystems $\mathrm{GmbH}$ ).

FJB staining. FJB staining was performed according to the protocols provided by the manufacturer (EMD Millipore). After incubating with $1 \%$ sodium hydroxide in $80 \%$ alcohol for $5 \mathrm{~min}$ and $70 \%$ alcohol for $2 \mathrm{~min}$, the cryosectioned samples 


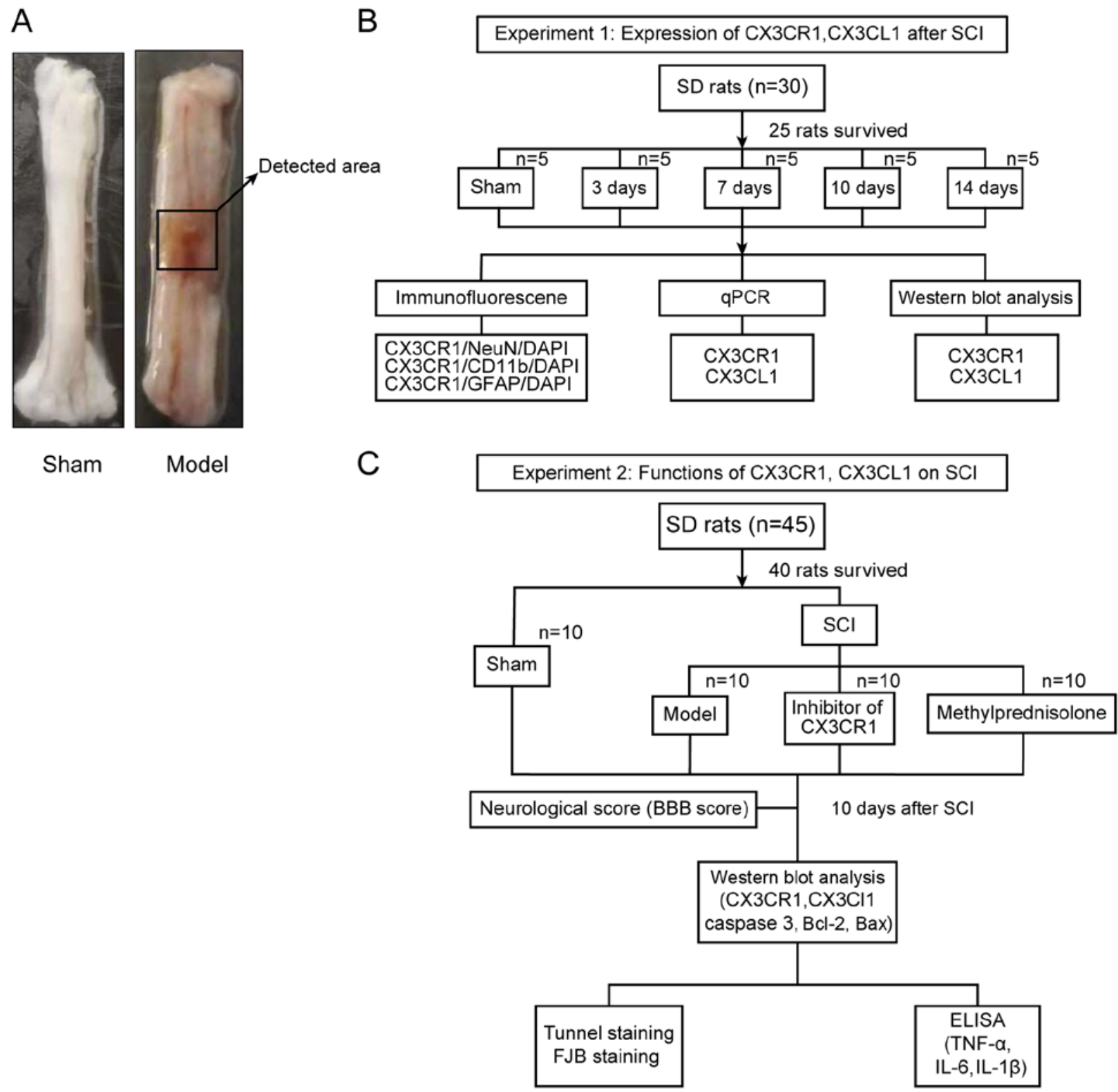

Figure 1. Study design. (A) The representative areas obtained from the injured rat spinal cords for further analysis. (B) Experiment subset I was employed for the time course expression analyses of CX3CL1/CX3CR1 after SCI. (C) Experiment subset II was employed to establish the functional role of CX3CL1/CX3CR1 signaling in SCI rats. SCI, spinal cord injury; CX3CL1, C-X3-C motif chemokine ligand 1CX3CR1, C-X3-C motif chemokine receptor 1; SD, Sprague-Dawley; q, quantitative; IL, interleukin; TNF, tumor necrosis factor; BBB, Basso Beattie Bresnahan; TUNEL, terminal deoxynucleotidyl transferase-mediated dUTP nick-end labeling; FJB, fluoro jade B; DAPI, 4',6-diamidino-2-phenylindole.

were then transferred to $0.06 \%$ potassium permanganate for $10 \mathrm{~min}$. Afterwards, they were immersed (room temperature) in $0.0004 \%$ fluoro-jade dye solution containing $0.1 \%$ acetic acid for $20 \mathrm{~min}$, followed by rinsing with deionized water. Then, they were dried in an oven at $50^{\circ} \mathrm{C}$ for $5-8 \mathrm{~min}$. The sections were then immersed in xylene for at least $1 \mathrm{~min}$ and then mounted with a non-aqueous and non-fluorescent plastic mounting medium, distyreneplasticiser xylene. The samples were observed under a Leica DMi8 confocal microscope and the images were captured using LASX software (Leica Microsystems, $\mathrm{GmbH}$ ).

TUNEL staining. Apoptosis was detected using TUNEL staining according to the manufacturer's protocol (Abcam). Frozen injured spinal cord tissue sections were soaked in $4 \%$ polyformaldehyde for $15 \mathrm{~min}$ and then shifted into a protease $\mathrm{K}$ working solution and incubated for $5 \mathrm{~min}$. The samples were immersed in $4 \%$ polyformaldehyde for another $5 \mathrm{~min}$ and rinsed with wash buffer twice for $5 \mathrm{~min}$ each. All the slices were incubated $\left(37^{\circ} \mathrm{C}\right)$ in DNA labeling solution and stored in a wet box away from light for $1 \mathrm{~h}$. After washing the slices, antibody solution was added and kept in a dark and wet box for $30 \mathrm{~min}$. The samples were then rinsed with deionizing solution for $5 \mathrm{~min}$. After air drying, they were then sealed with DAPI (room temperature in the dark for $30 \mathrm{~min}$ ). All the slides were observed under a laser confocal microscope Leica DMi8 (Leica Microsystems, GmbH) and images were captured using the LASX software.

ELISA. The concentrations of interleukin-1 $\beta$ (IL-1 $\beta$ ), tumor necrosis factor- $\alpha$ (TNF- $\alpha$ ) and IL-6 in the serially collected 
Table I. Assessment of locomotive recovery of rats from sham, injury, inhibitor, or methylprednisolone administration groups 10 days after operation via BBB scoring.

\begin{tabular}{lcccc}
\hline & \multicolumn{3}{c}{ Group } \\
\cline { 2 - 5 } $\begin{array}{l}\text { BBB score } \\
\text { and difference }\end{array}$ & Sham & Model & Inhibitor & Me \\
\hline BBB score & $19.30 \pm 0.95$ & $5.40 \pm 0.84$ & $7.70 \pm 0.82$ & $7.40 \pm 0.70$ \\
P-value & $<0.001^{\mathrm{a}}$ & $<0.001^{\mathrm{a}}$ & $<0.001^{\mathrm{a}}$ \\
P-value & & $<0.001^{\mathrm{b}}$ & $<0.001^{\mathrm{b}}$ \\
P-value & & $0.391^{\mathrm{c}}$ & \\
\hline
\end{tabular}

${ }^{a} \mathrm{P}<0.05$ vs. the sham group, ${ }^{\mathrm{b}} \mathrm{P}<0.05$ vs. the model group; ${ }^{\mathrm{C}} \mathrm{P}<0.05$ vs. the Me group. Me, methylprednisolone; $\mathrm{BBB}$, Basso Beattie Bresnahan.

serum samples were measured on the 10th postoperative day using the ELISA kits for IL-1 $\beta$ (cat. no. MK1198); TNF- $\alpha$ (cat. no. EK0526); and IL-6 (cat. no. EK0412) (all from Wuhan Boster Biological Technology, Ltd.). The serum was obtained by centrifugation $\left(5,700 \times \mathrm{g}\right.$, for $10 \mathrm{~min}$ and $\left.4^{\circ} \mathrm{C}\right)$ after the blood was kept overnight at room temperature. After preparation, the sample and standard (100 $\mu 1$ each) were added to a 96-well plate and incubated at $37^{\circ} \mathrm{C}$ for $90 \mathrm{~min}$. Then, the antibodies were labeled with biotin in $100 \mu \mathrm{l}$ aliquots in each well and reacted for $60 \mathrm{~min}$ at $37^{\circ} \mathrm{C}$. Then samples were rinsed with $0.01 \mathrm{M}$ TBS and $100 \mu 1$ avidin-biotin complex was added to each well and allowed to react at $37^{\circ} \mathrm{C}$ for $30 \mathrm{~min}$, followed by 5 rinses with $0.01 \mathrm{M}$ TBS. The reaction time was $<30 \mathrm{~min}$, after adding tetramethyl benzidine (TMB) at $37^{\circ} \mathrm{C}$ without light and then TMB termination solution was added. Using a microplate reader, the absorbance at $450 \mathrm{~nm}$ was measured and the protein concentrations $(\mathrm{ng} / \mathrm{ml})$ were calculated using standard curves.

Statistical analysis. All the collected data were analyzed using SPSS 22.0 software (IBM, Corps.). A one-way analysis of variance was employed for multiple comparisons among different groups and a Student's t-test was employed to compare the results between two groups, given that all data was normally distributed. The data were presented as the mean \pm standard deviation, Tukey honest significant difference test was used as a post hoc test. $\mathrm{P}<0.05$ was considered to indicate a statistically significant difference, while a $\mathrm{P}<0.01$ was considered as indicative of a high level of significance.

\section{Results}

CX3CR1 and CX3CL1 are upregulated at both the MRNA and protein levels in the injured spinal cords after SCI. CX3CL1, also known as fractalkine, is a chemokine that is uniquely anchored to the plasma membrane. The CX3CL1/CX3CR1 axis has also been frequently described for its role in the pathogenesis and progression of numerous CNS diseases and injuries. Thus, in order to evaluate the time course of changing CX3CL1/CX3CR1 signaling, samples were collected at different time points after SCI and assessed accordingly. CX3CR1, at both the mRNA and protein levels, increased after 3 days of SCI, reached its peak on the 10th day, and decreased significantly on the 14th day (Fig. 2B and D). These results indicate that CX3CR1 expression increased, mediating a local inflammatory response and decreased significantly 14 days after SCI. Similarly, the expression of CX3CL1 at the mRNA level decreased significantly on the 3rd day, then increased gradually, reaching its peak on the 10th day and decreasing significantly on the 14th day after SCI (Fig. 2A). These results suggest that CX3CL1 exists as a membrane-bound as well as a secretory protein. After SCI, the expression of CX3CL1 decreased for a short period of time, potentially due to local injury and being released into the blood as a chemokine. It then gradually increased, possibly due to enhanced expression, adhesion and aggregation. Its expression then again decreased following a constant pattern, indicative of a relatively stable level of protein on the 14th post-SCI day.

CX3CR1 is upregulated in microglia, astrocytes and neurons after SCI. In order to determine the specific cell types implicated in CX3CL1/CX3CR1 signaling, immunofluorescence was performed to identify certain cell types expressing CX3CR1 among neurons, microglia and astrocytes. The results show that CX3CR1 was not detectable in neurons at all (Fig. 3A). In contrast, both microglia and astrocytes displayed the expression of CX3CR1 at the protein level (Fig. 3B and D). Furthermore, they were upregulated significantly after SCI, especially in the microglia, suggesting a major role of microglia following SCI (Fig. 3C).

AZD8797 treatment enhances the early behavioral recovery after SCI. Considering extensive involvement of CX3CL1/CX3CR1 signaling in CNS diseases and injuries, the effect of AZD8797 treatment on SCI was yet to be examined, and, hence, evaluation of locomotive recovery via BBB scoring was performed on the 10th day after the operation. As shown in Table I, the BBB score was reduced nearly 4 times in the SCI group compared with the sham control. In contrast, the treatment with AZD8797 significantly improved the BBB score when compared to the untreated SCI group. Similarly, treatment with methylprednisolone also significantly improved the BBB score. However, there was no statistical difference between the BBB scores in the AZD8797 treated group and the methylprednisolone treated group. Thus, the results clearly indicate that AZD8797 treatment enhances early behavioral recovery after SCI and is as effective as the methylprednisolone treatment. 
A

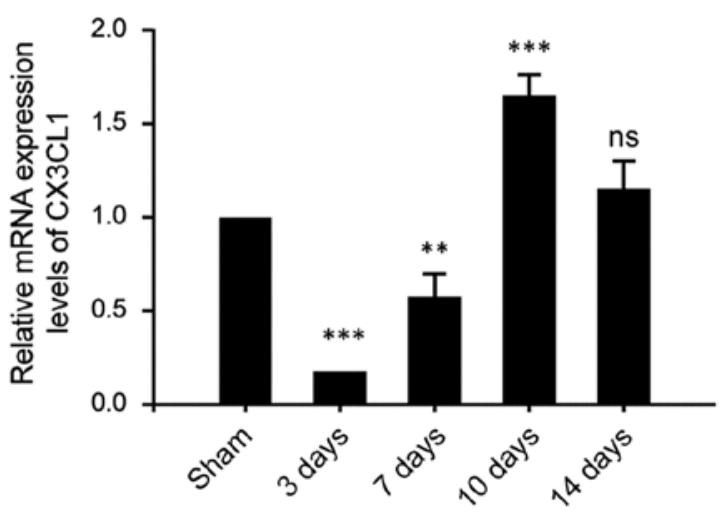

C
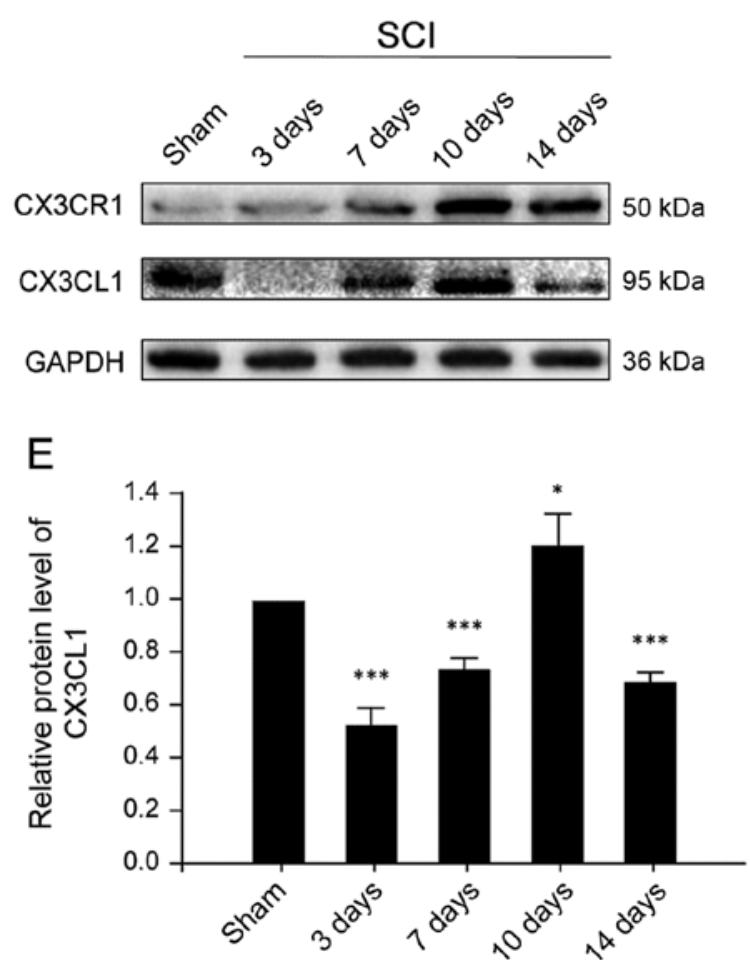

B
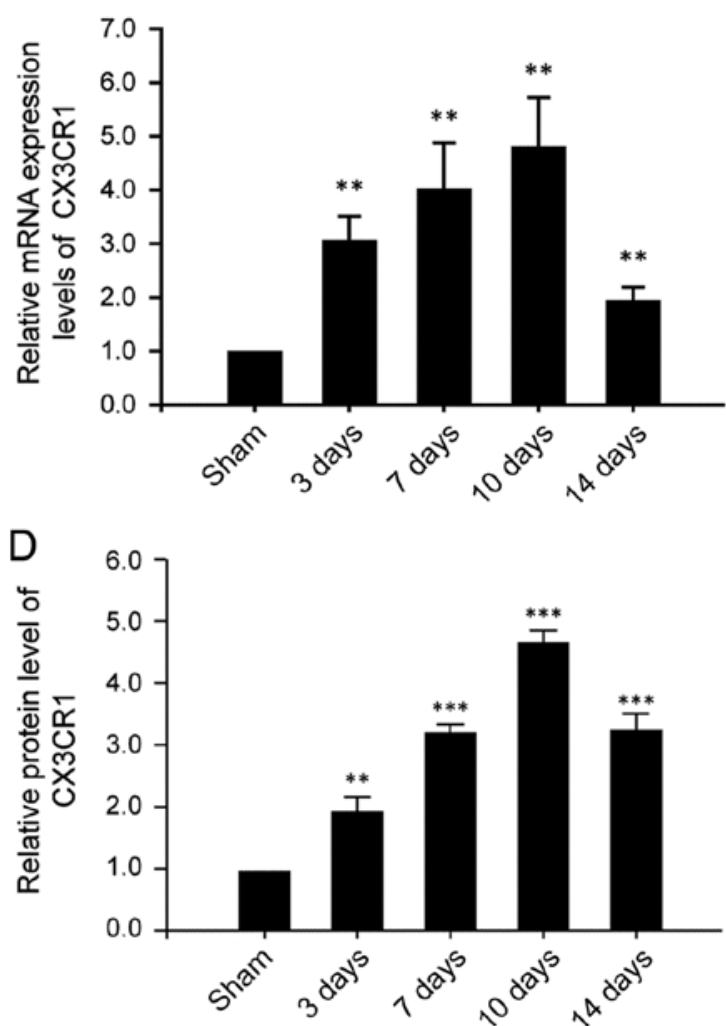

Figure 2. Time course oftheexpression of CX3CL1/CX3CR1 in the spinal cord at both the mRNA and protein levels after SCI. (A) CX3CL1/(B) CX3CR1 mRNA levels after SCI in rat spinal cord tissue obtained at each time point were analyzed by quantitative PCR. Quantitative analyses were then performed for assessing the relative quantity normalized to the internal control and then further normalized to the mean value of the sham group. The data was presented as the mean $\pm \mathrm{SD} .{ }^{* *} \mathrm{P}<0.01$ and ${ }^{* * *} \mathrm{P}<0.001$ vs. sham; ${ }^{\mathrm{ns}} \mathrm{P}>0.05$ vs. sham. $\mathrm{n}=5$. (C) The representative examples of the western blot analysis of the CX3CL1/CX3CR1 protein levels in the spinal cord obtained at each time pointafter SCI. Quantitative analysis of the western blot result of (D) CX3CL1/(E) CX3CR1 using ImageJ software, followed by normalizing the mean values to the sham group for (D) CX3CR1 and (E) CX3CL1. The data was represented as the mean \pm SD. " $\mathrm{P}<0.05$, ${ }^{* *} \mathrm{P}<0.01$ and ${ }^{* * *} \mathrm{P}<0.001$ vs. sham; $\mathrm{n}=5$. SD, standard deviation; SCI, spinal cord injury; CX3CL1, C-X3-C motif chemokine ligand 1CX3CR1, C-X3-C motif chemokine receptor 1 ; ns, not significant.

AZD8797 treatment suppresses the activation of apoptosis machinery after SCI. To explore the underlying mechanism for CX3CR1 inhibitor-mediated enhanced behavioral recovery, the expression levels of apoptosis-related molecules, including caspase $3, \mathrm{Bcl}-2$ and Bax were evaluated, in addition to the expression levels of CX3CL1/CX3CR1 signaling after AZD8797 treatment. Cleaved caspase 3 has two molecular weight forms of 17 and $19 \mathrm{kDa}$. In the present experiment, cleaved caspase 3 with the weight form of $17 \mathrm{kDa}$ was tested as the expression of cleaved caspase 3 with the molecular weight form of $19 \mathrm{kDa}$ was weak (Fig. 4A). The total caspase 3 in each group is equivalent to that of the reference $\beta$-actin, so the ratio between cleaved caspase 3 and $\beta$-actin was calculated to reflect apoptosis. For the CX3CR1 level, the results showed that there was a significant increase in the SCI model group compared with the sham control $(\mathrm{P}<0.001)$. However, treatment with AZD8797 $(\mathrm{P}=0.861)$ or with methylprednisolone $(\mathrm{P}=0.771)$ did not result in a significant change in CX3CR1 levels (Fig. 4A and C). Comparatively, CX3CL1 levels were significantly elevated in the SCI model group when compared with the sham control $(\mathrm{P}<0.001)$. AZD8797 treatment resulted in a significant increase in CX3CL1 levels when compared to the untreated model $(\mathrm{P}=0.012)$ and to the methylprednisolone treated group $(\mathrm{P}=0.007)$. In contrast, 


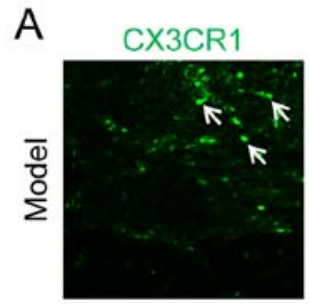

B CX3CR1
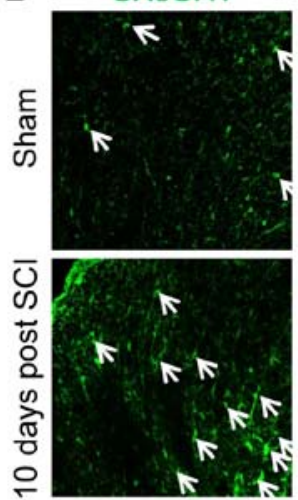

D
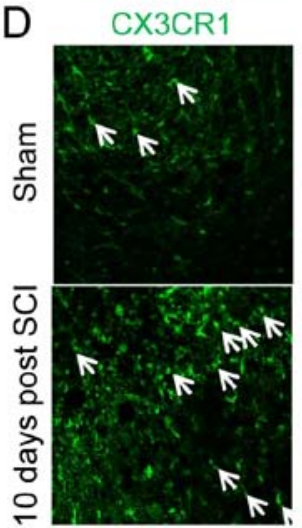

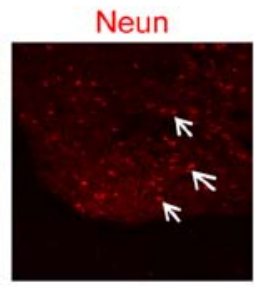

CD11-b
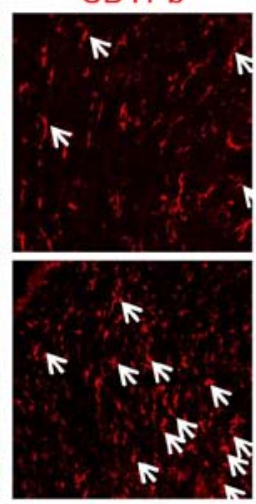

GFAP

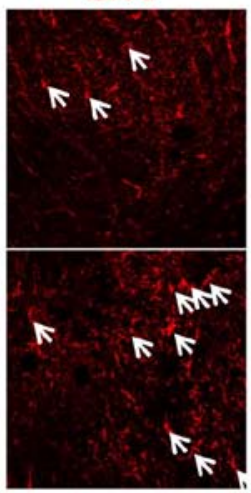

DAPI

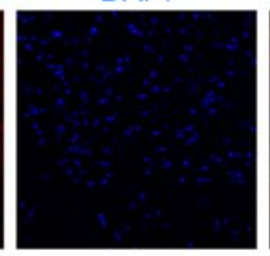

DAPI
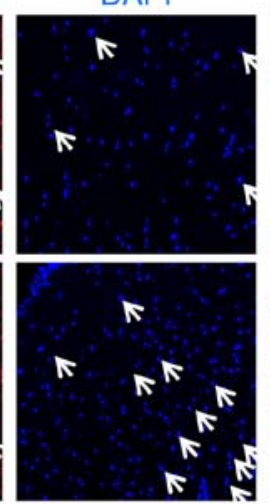

DAPI

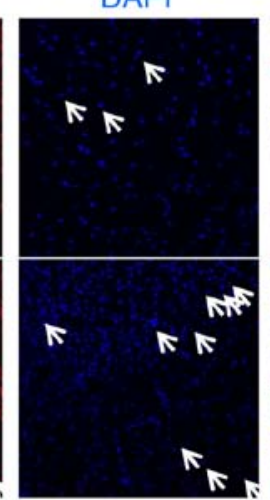

Merge

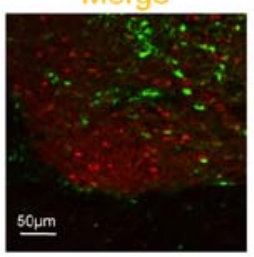

Merge

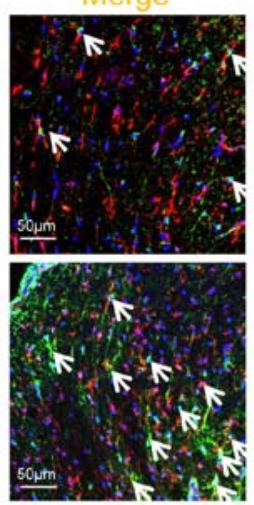

Merge

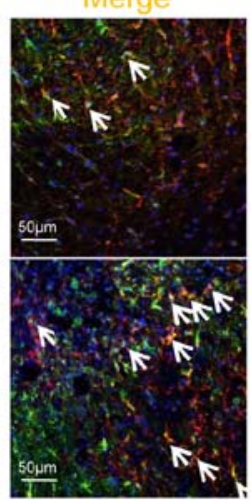

C

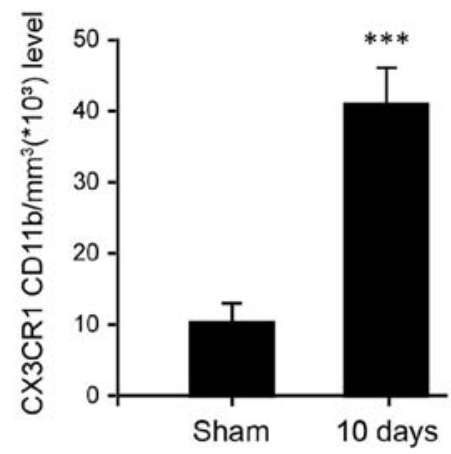

$E$

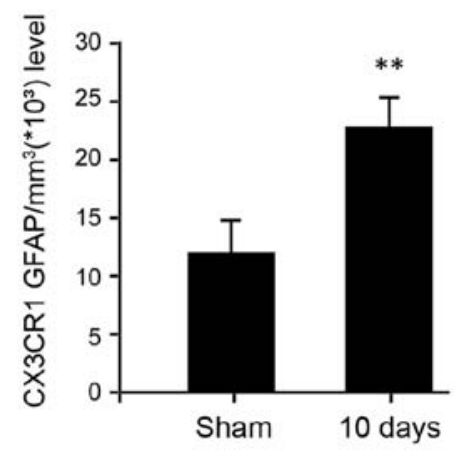

Figure 3. Fluorescence immunostaining for cellular localization of CX3CR1 in the injured spinal cord after SCI. (A) Co-immunostaining of CX3CR1 (green) and the neuronal marker NeuN (red). Note that CX3CR1 was not localized in the NeuN positive cells (neurons). (B) Co-immunostaining of CX3CR1 (green) and the microglial marker CD11-B (red) after SCI. The arrows indicate the co-localization (yellow) of CX3CR1 and CD11-B. (C) The corresponding quantitative analysis is shown. (D) Co-immunostaining of CX3CR1 (green) and the astrocyte marker GFAP (red) in injured spinal cord tissues. The arrows indicate the co-localization (yellow) of CX3CR1 and GFAP. The corresponding quantitative analysis is shown in (E). The data was represented as the mean \pm SD. ${ }^{* *} \mathrm{P}<0.01$ and ${ }^{* * *} \mathrm{P}<0.001$ vs. sham. Scale bar $=50 \mu \mathrm{m}$. $\mathrm{n}=5$. Me, methylprednisolone; ns, not significant; GFAP, glial fibrillary acidic protein; SCI, spinal cord injury; CX3CL1, C-X3-C motif chemokine ligand 1CX3CR1, C-X3-C motif chemokine receptor 1; DAPI, 4',6-diamidino-2-phenylindole; CD, cluster of differentiation.

methylprednisolone treatment did not have any effect on CX3CL1 levels when compared to the untreated model group $(\mathrm{P}=0.643$; Fig. 4A and B).

For the apoptosis machinery-related molecules, including caspase 3, Bax and $\mathrm{Bcl}-2$, the results consistently show significant differences between the SCI model and the sham control ( $\mathrm{P}<0.001$; Fig. 4D-F). Treatment with AZD8797 or methylprednisolone significantly reversed the changes when compared to the untreated SCI group ( $<<0.01$; Fig. 4D-F). Treatment with AZD8797 was even more effective in reducing the elevated levels of Bax in the SCI model when compared to the methylprednisolone treatment $(\mathrm{P}=0.029$; Fig. 4F), but, for Bcl-2 and caspase 3, treatment with AZD8797 was as effective as that with methylprednisolone $(\mathrm{P}=0.158$; Fig. $4 \mathrm{D} ; \mathrm{P}=0.586$; Fig. 4E). Accordingly, given the activation of apoptosis machinery after SCI through the CX3CL1/CX3CR1 signaling pathway, significantly enhanced cell necrosis was also noted in the SCI model compared to the sham control $(\mathrm{P}<0.001$; Fig. 5A and B). AZD8797 treatment significantly reduced cell necrosis when compared to the untreated SCI group $(\mathrm{P}<0.01)$. The methylprednisolone treatment also reduced necrosis when compared to the untreated SCI group $(\mathrm{P}<0.01)$ but was not as effective as the AZD8797 treatment $(\mathrm{P}<0.05)$. Note that the Bax level was also different between these two groups (Fig. 4F).

Intriguingly, the pattern of neuronal death caused by apoptosis was consistent with the evaluation for neuronal necrosis (Fig. 5C and D). Overall, these results have demonstrated that AZD8797 treatment suppresses the activation of apoptosis machinery following SCI.

AZD8797 treatment inhibits the inflammatory response after $S C I$. Due to the widespread influence of CX3CL1/CX3CR1 signaling during the inflammatory response, the inhibition of 
A

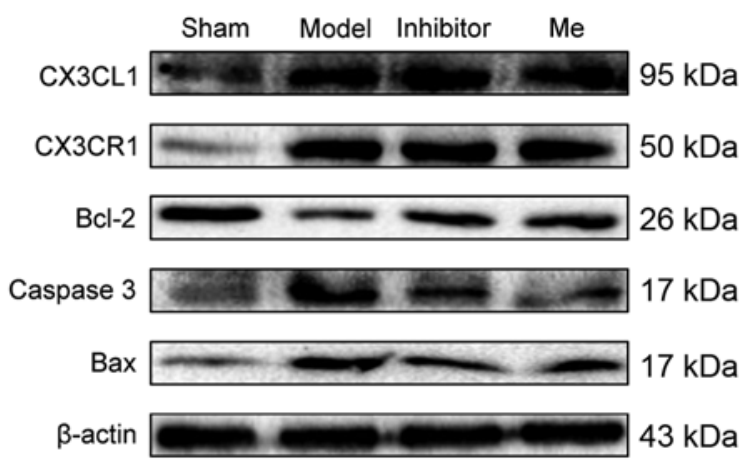

B

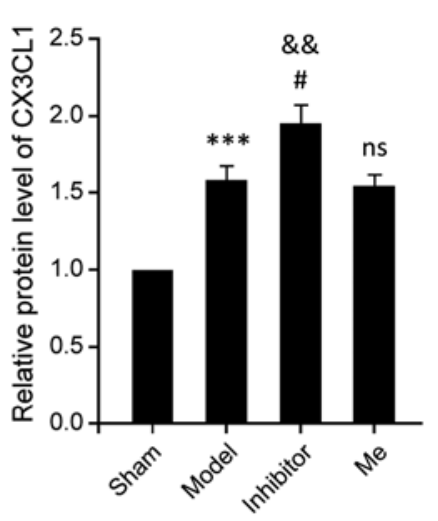

C

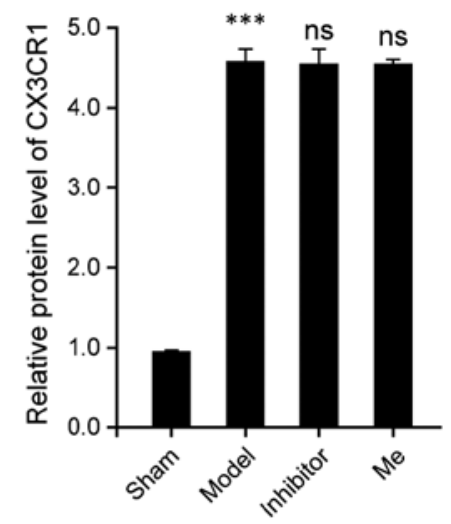

D

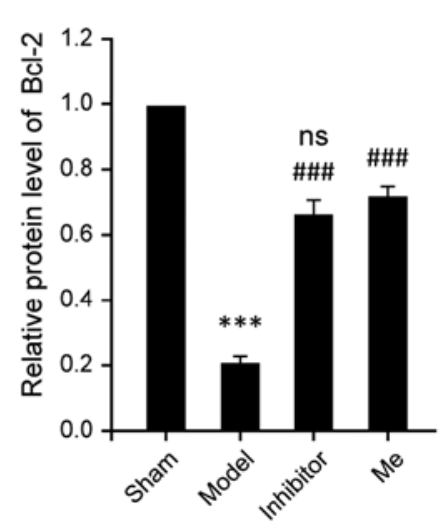

E

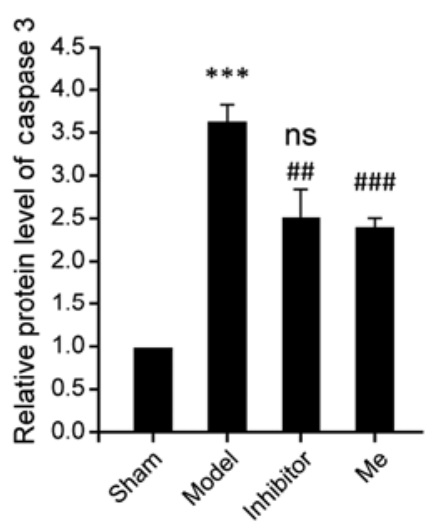

$\mathrm{F}$

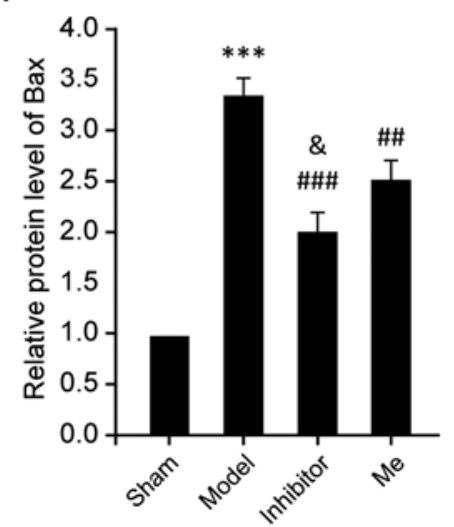

Figure 4. AZD8797 reduces neuronal apoptosis after rat SCI. (A) The representative examples of the western blot analysis results for the expression of CX3CL1, CX3CR1, caspase 3, Bcl-2 and Bax in four groups as indicated 10 days after SCI. (B) Quantitative analysis of (B) CX3CL1, (C) CX3CR1, (D) Bcl2, (E) caspase 3 and (F) Bax expression in the western blotting in the sham, Model, Inhibitor and Me groups 10 days after SCI. Data is presented as means \pm standard deviation. ${ }^{* * *} \mathrm{P}<0.001$ vs. sham, ${ }^{n s} \mathrm{P}>0.05$ vs. Injury, ${ }^{~} \mathrm{P}<0.05$ and ${ }^{\& \&} \mathrm{P}<0.01$ vs. Me. ${ }^{\#} \mathrm{P}<0.05,{ }^{\# \#} \mathrm{P}<0.01$ and ${ }^{\# \# \#} \mathrm{P}<0.001$ vs. Injury. $\mathrm{n}=10$. Me, methylprednisolone; ns, not significant; GFAP, glial fibrillary acidic protein; SCI, spinal cord injury; CX3CL1, C-X3-C motif chemokine ligand 1CX3CR1, C-X3-C motif chemokine receptor 1.

this pathway by AZD8797 could suppress numerous types of chemokines and cytokines. Thus, the serum levels of multiple cytokines (e.g., IL-1 $\beta$, IL- 6 and TNF- $\alpha$ ) needed to be determined. As shown in Fig. 6, on the 10th postoperative day, the serum levels of these three cytokines were significantly increased in the SCI group compared to the sham control. However, the SCI treated with AZD8797 resulted in a significant reduction of the elevated levels for these three cytokines when compared with the SCI group without treatment. Methylprednisolone treatment resulted in a similar reduction in TNF- $\alpha$ and IL-6 when compared to the AZD8797-treated group, but with a slightly smaller, yet significant, reduction of IL-1 $\beta$ when compared to the AZD8797-treated group. As regards the potential mechanisms of the CX3CR1-mediated inflammatory response after SCI, microglia and astrocytes play an important role (Fig. 7).

Therefore, these results suggest that this particular CX3CR1 inhibitor can suppress the overall inflammatory response after SCI and it is as effective as, or even better than, methylprednisolone.

\section{Discussion}

Currently, the precise role of the fractalkine/CX3CR1 signaling pathway following SCI remains largely unknown.
To this end, using an SCI rat model, the association of the CX3CL1/CX3CR1 signaling pathway with inflammation was explored, as well as necrosis after SCI. Combining evaluations for the expression of both CX3CL1 and CX3CR1 at both the mRNA and protein levels, it was found that CX3CR1 and CX3CL1 expression levels consistently increase after 3 days of SCI, reaching a peak after 10 days, and decrease after 14 days. This pattern of short-term alteration is consistent with its role as a chemokine during the inflammatory response after SCI. This pattern also indicates a gradual increase in its role of adhesion and aggregation, whereas a stable decrease in its expression following 14 days matches the short-term release of most chemokines. Interestingly, the suppression of the CX3CL1/CX3CR1 signaling pathway by AZD8797 led to improved recovery after SCI as shown by BBB scoring, compared with the sham group within the same period. However, this CX3CR1 inhibitor did not change the expression level of the CX3CR1 protein but enhanced the expression level of the CX3CL1 protein. AZD8797 is a non-competitive inhibitor for CX3CR1. The above result suggests that it has no influence on the CX3CR1 protein itself except for affecting the activation of its downstream signaling pathway. More intriguingly, AZD8797 increases the expression level of CX3CL1, indicating that there is a feedback loop, probably a compensatory effect after blocking the signaling pathway, 
A

Sham

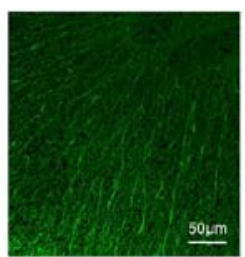

C
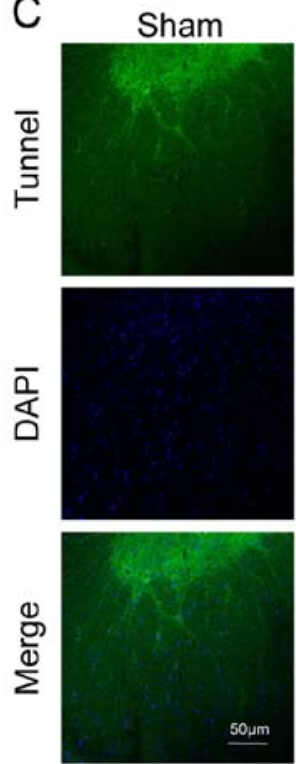

Model

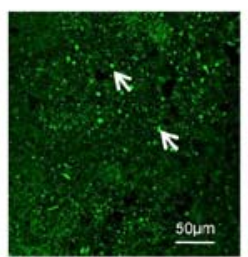

Model
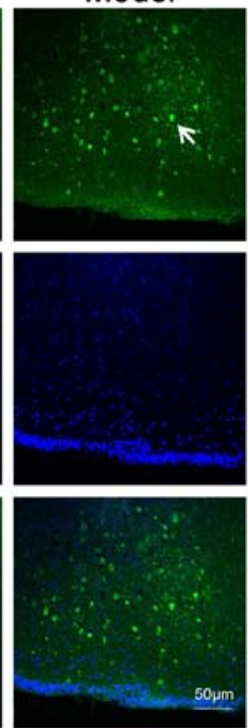

Inhibitor

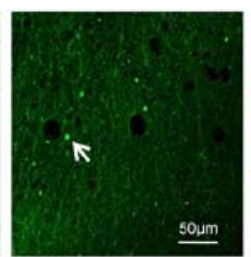

Inbibitor
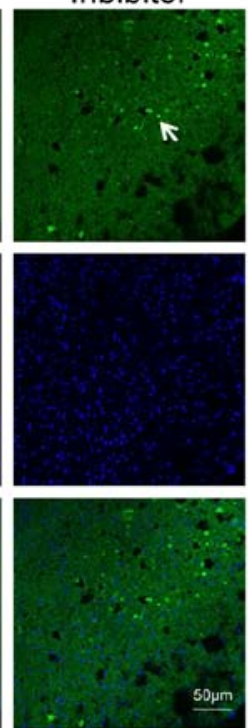

$\mathrm{Me}$

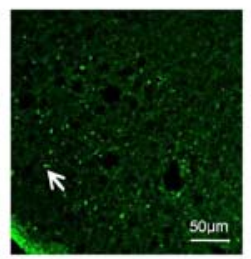

$\mathrm{Me}$
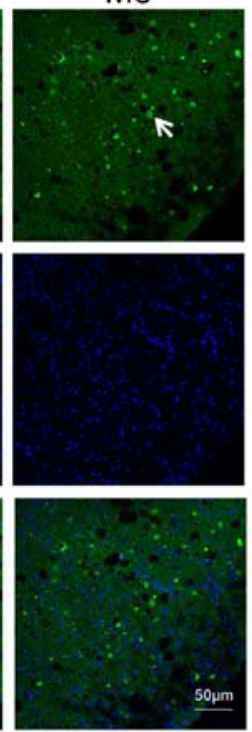

B

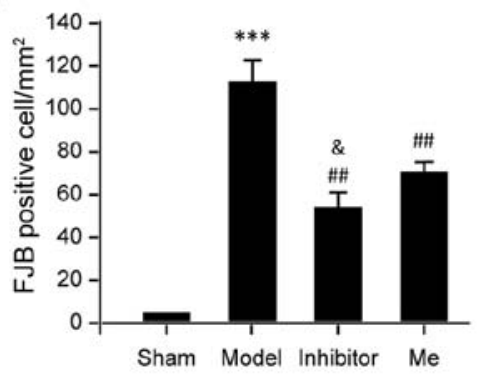

D

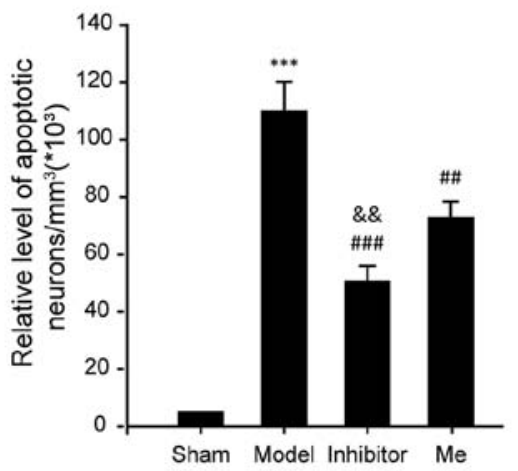

Figure 5. Evaluation of neuronal death. (A) Neuronal death was examined by FJB staining. FJB-positive (green) cells per mm ${ }^{2}$ were quantified accordingly. (B) The data was represented as mean $\pm \mathrm{SD}$. ${ }^{* * *} \mathrm{P}<0.01$ vs. sham; ${ }^{\# \#} \mathrm{P}<0.01$ vs. Injury; and ${ }^{\circledR} \mathrm{P}<0.05$ vs. Me. Scale bar, $50 \mu \mathrm{m}$. $\mathrm{n}=10$. (C) Neuronal apoptosis was examined by a TUNEL assay using immunofluorescence. In each section, apoptotic neurons were labeled by TUNEL (green), while nuclei were labeled with DAPI (blue). TUNEL-nuclei positive neurons per $\mathrm{mm}^{3}$ were (D) quantified accordingly. The data are presented as the mean \pm SD. White arrows indicate positive signals. ${ }^{* * *} \mathrm{P}<0.01$ vs. sham; ${ }^{\# \#} \mathrm{P}<0.01$ and ${ }^{\# \# \#} \mathrm{P}<0.001$ vs. injury; and ${ }^{\& \&} \mathrm{P}<0.01$ vs. Me. Scale bar, $50 \mu \mathrm{m}$. $\mathrm{n}=10$. TUNEL, terminal deoxynucleotidyl transferase-mediated dUTP nick-end labeling; FJB, fluoro jade B; DAPI, 4',6-diamidino-2-phenylindole; SD, standard deviation; SCI, spinal cord injury; CX3CL1, C-X3-C motif chemokine ligand 1CX3CR1, C-X3-C motif chemokine receptor 1; Me, methylprednisolone.

A

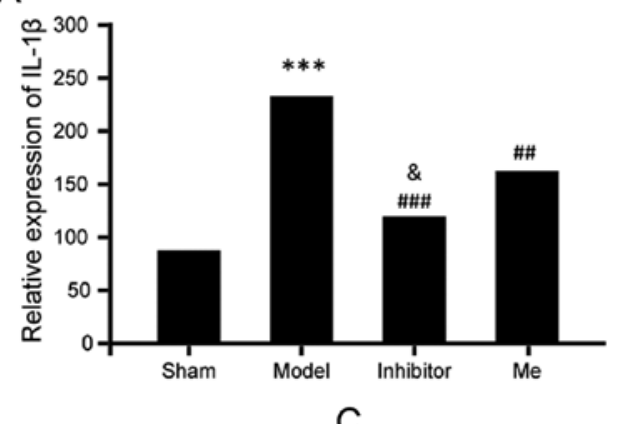

B

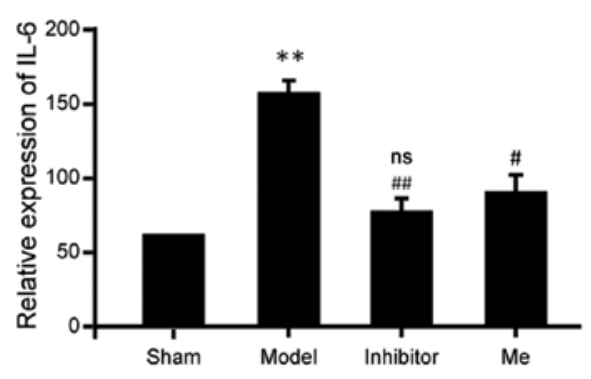

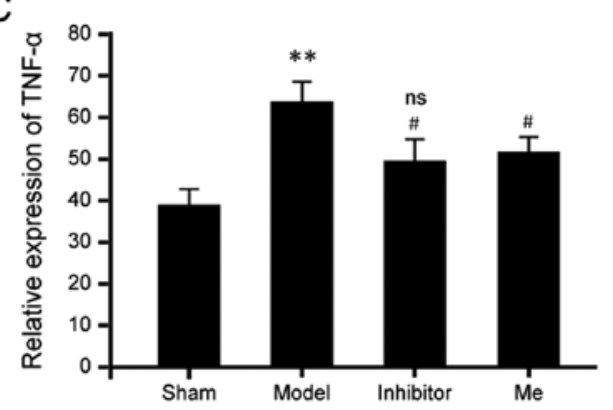

Figure 6. Changes of the serum concentrations of IL-1 $\beta$, IL- 6 and TNF- $\alpha$ after SCI injury in treated and untreated groups. The three inflammatory cytokines (A) IL-1 $\beta$, (B) IL-6 and (C) TNF- $\alpha$ were measured by ELISA. Their concentrations are normalized to the mean values of the sham group. The data was represented as the mean $\pm \mathrm{SD} .{ }^{* *} \mathrm{P}<0.01$ vs. sham; ${ }^{* * *} \mathrm{P}<0.001$ vs. sham; ${ }^{\#} \mathrm{P}<0.05,{ }^{\# \#} \mathrm{P}<0.01$ and ${ }^{\# \# t} \mathrm{P}<0.001$ vs. injury; ${ }^{\text {ns }} \mathrm{P}>0.05$ vs. Me; and ${ }^{\&} \mathrm{P}<0.05$ vs. Me. $\mathrm{N}=10$. IL, interleukin; TNF, tumor necrosis factor; SCI, spinal cord injury. 


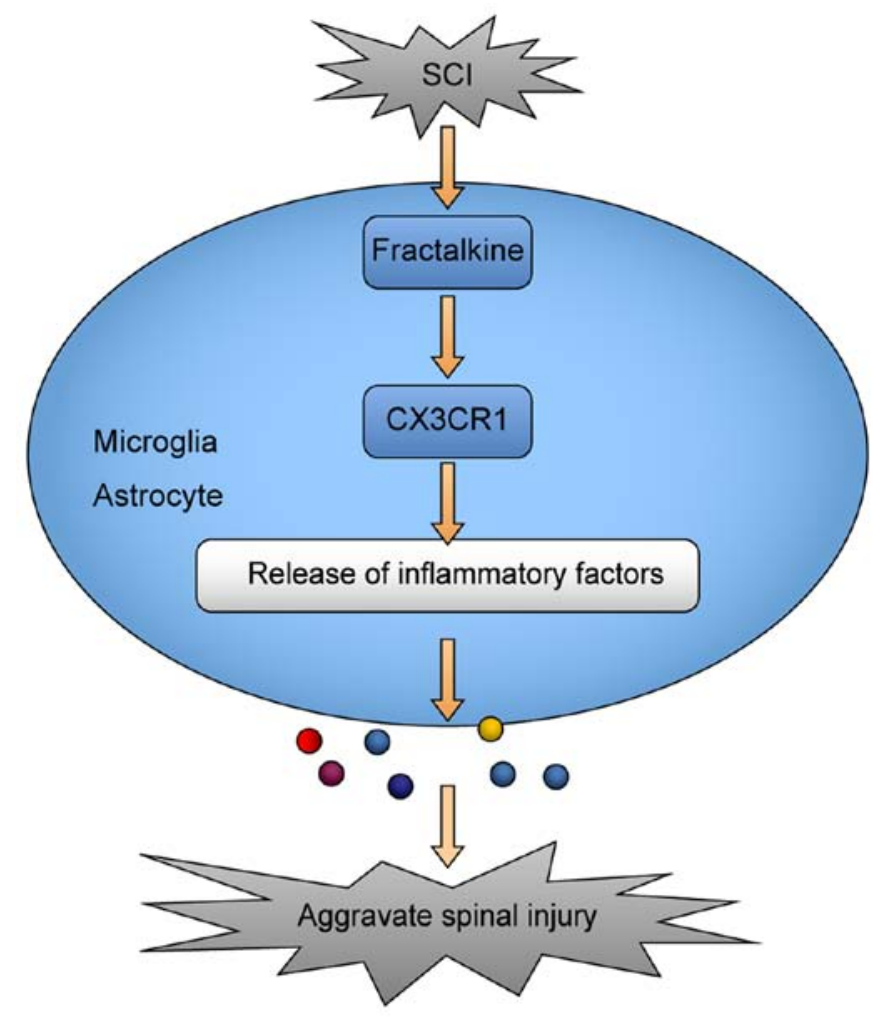

Figure 7. Potential mechanisms for the CX3CR1-mediated effects after SCI. Briefly, SCI induces a rapid release of fractalkine (CX3CL1) and then, with the adhesion and aggregation of CX3CL1, the expression of CX3CR1 increased in both microglia and astrocytes, causing the release of inflammatory factors, which later aggravates the severity of spinal injury. CX3CL1, C-X3-C motif chemokine ligand 1CX3CR1, C-X3-C motif chemokine receptor 1 ; SCI, spinal cord injury.

which has been pointed out previously but is yet to be further defined (40).

Unlike CNS diseases in most cases, SCI creates a short term but usually drastic opening of the blood-brain barrier. It certainly cannot be ignored that CX3CL1 functions as an adhesion molecule in a subset of leukocytes, that allows these cells to cross the blood vessel wall by binding to CX3CR1 expressed in vascular endothelial cells (41). Some clinical trials have demonstrated that, in cases of ischemic stroke, endovascular therapy is effective, especially for those caused by a proximal intracranial occlusion within the anterior circulation (42-44). Given that the short-term alteration pattern of CX3CL1/CX3CR1 signaling matches the time window of reactive astrogliosis $(45,46)$, microglia, known as resident immune cells of the CNS, are thus considered to generally play a major role in inflammatory responses in the CNS, following either CNS injuries or diseases. Specifically, microglia express CX3CR1 at a high level and are an activated cell type detectable through phenotypic transformation following ischemia $(47,48)$. The deficiency in microglial CX3CR1 can cause communication defects among neurons, microglia and astrocytes during numerous CNS diseases or even injuries (49). In addition, CX3CL1 activation is correlated with the specific type of neuropathic pain induced by multiple sclerosis through interaction with CX3CR1 (50). Moreover, the levels of CX3CL1 are potentially regulated by diverse neurotoxic stimuli and its signaling is correlated with several types of
CNS diseases, including HIV infection, epilepsy, and cerebral tumors among other neuropathologies (51).

Methylprednisolone is a glucocorticosteroid commonly used in the clinic with strong immunosuppressive and anti-inflammatory effects. A phase III clinical study (52-53) of the National Association of Acute Spinal Cord Injury Study has confirmed that it is effective to use high-dose methylprednisolone to treat the early stage of acute SCI. The known mechanisms for methylprednisolone's effect in the treatment of SCI include i) inhibition of lipid peroxidation, apoptosis, inflammation and the release of inflammatory substances, and improvement of spinal microcirculation, ii) modification of vascular permeability and tissue edema, and iii) inhibition of excitatory amino acid toxicity, promotion of neurotrophic factors and the release of other cytokines. However, the detailed molecular mechanism behind methylprednisolone's effect is not clear. Notably, previous studies demonstrated that in vitro lipopolysaccharide- and interferon $\gamma$-induced release of multiple cytokines (i.e., IL-1 $\beta, \mathrm{TNF} \alpha$, and IL-6) in cultured microglia can be effectively suppressed through the activity of CX3CL1 signaling. This suggests that CX3CL1/CX3CR1 signaling is vital in modulating upstream production and cytokine release from microglia (51), thereby contributing to the feedback loop as well. By inhibiting the cx3cl1/CX3CR1 pathway, AZD8797 can suppress the role of microglia and astrocytes, thus preventing the development of the inflammatory response $(54,55)$. The present study shows that blocking the CX3CR1 signaling with AZD8797 results in, not only a more effectively reduced concentration of the inflammatory cytokine, IL-1 $\beta$ (which is associated with a better recovery after injury), but also lower apoptosis and necrosis levels when compared to methylprednisolone treatment.

In conclusion, the present study has demonstrated that treatment with the CX3CR1 specific inhibitor, AZD8797, can facilitate the recovery of neurological function in the acute phase of SCI through suppression of CX3CL1/CX3CR1 signaling. The current findings provide a novel, practical approach for SCI treatment. Nevertheless, further investigation is required for elucidating the combined effect of CX3CL1/CX3CR1 signaling, especially considering its extensive involvement and potential side effects, as well as analyzing its efficacy and safety in preclinical and clinical trials.

\section{Acknowledgements}

Not applicable.

\section{Funding}

No funding was received.

\section{Availability of data and materials}

The data used to support the findings of this study are available from the corresponding author upon request.

\section{Authors' contributions}

GC and ZZ constructed the SCI model, performed western blot analysis, reverse transcription-quantitative PCR and 
immunofluorescence analyses. WS, LW and FY determined the FJB staining. XY and XQ performed the TUNEL staining. ST and DL analyzed and interpreted the data. MW finished the assessment of locomotive recovery of rats (BBB score). GC revised the manuscript critically for important intellectual content. ST, DL, GC and ZZ designed the study, supervised the research group and gave final approval of the version to be published. The final version of the manuscript has been read and approved by all authors.

\section{Ethics approval and consent to participate}

The present study certifies that all applicable institutional and governmental regulations concerning the ethical use of animals were followed during the course of this research.

\section{Patient consent for publication}

Not applicable.

\section{Competing interests}

The authors have declared that they have no competing interests.

\section{References}

1. Hyder AA, Wunderlich CA, Puvanachandra P, Gururaj G and Kobusingye OC: The impact of traumatic brain injuries: A global perspective. NeuroRehabilitation 22: 341-353, 2007.

2. Lee BB, Cripps RA, Fitzharris M and Wing PC: The global map for traumatic spinal cord injury epidemiology: Update 2011, global incidence rate. Spinal Cord 52: 110-116, 2014.

3. Fitzharris M, Cripps RA and Lee BB: Estimating the global incidence of traumatic spinal cord injury. Spinal Cord 52: 117-122, 2014.

4. Oertel M, Kelly DF, McArthur D, Boscardin WJ, Glenn TC, Lee JH, Gravori T, Obukhov D, McBride DQ and Martin NA: Progressive hemorrhage after head trauma: Predictors and consequences of the evolving injury. J Neurosurg 96: 109-116, 2002.

5. Chen H, Guo Y, Chen SW, Wang G, Cao HL, Chen J, Gu Y and Tian HL: Progressive epidural hematoma in patients with head trauma: Incidence, outcome and risk factors. Emerg Med Int 2012: 134905, 2012.

6. Cuenca PJ, Tulley EB, Devita D and Stone A: Delayed traumatic spinal epidural hematoma with spontaneous resolution of symptoms. J Emerg Med 27: 37-41, 2004.

7. Khuyagbaatar B, Kim K and Kim YH: Effect of bone fragment impact velocity on biomechanical parameters related to spinal cord injury: A finite element study. J Biomech 47: 2820-2825, 2014.

8. Persson C, McLure S, Summers J and Hall R: The effect of bone fragment size and cerebrospinal fluid on spinal cord deformation during trauma: An ex vivo study. J Neurosurg Spine 10: 315-323, 2009.

9. Wilcox RK, Boerger TO, Allen DJ, Barton DC, Limb D, Dickson RA and Hall RM: A dynamic study of thoracolumbar burst fractures. J Bone Joint Surg Am 85: 2184-2189, 2003.

10. Scholten AC, Haagsma JA, Panneman MJM, Beeck EF, Van and Suzanne P: Traumatic brain injury in the Netherlands: Incidence, costs and disability-adjusted life years. PLoS One 9: e110905, 2014.

11. García-Altés A, Pérez K, Novoa A, Suelves JM, Bernabeu M, Vidal J, Arrufat V, Santamariña-Rubio E, Ferrando J, Cogollos M, et al: Spinal cord injury and traumatic brain injury: A cost-of-illness study. Neuroepidemiology 39: 103-108, 2012.

12. Guha A: Management of traumatic brain injury: Some current evidence and applications. Postgrad Med J 80: 650, 2004.

13. Borgens RB and Liu-Snyder P: Understanding secondary injury. Q Rev Biol 87: 89-127, 2012.
14. Oyinbo CA: Secondary injury mechanisms in traumatic spinal cord injury: A nugget of this multiply cascade. Acta Neurobiol Exp (Wars) 71: 281-299, 2011.

15. Maas AIR, Nino S and Ross B: Moderate and severe traumatic brain injury in adults. Lancet Neurol 7: 728-741, 2008.

16. Silva NA, Sousa N, Rui LR and Salgado AJ: From basics to clinical: A comprehensive review on spinal cord injury. Prog Neurobiol 114: 25-57, 2014.

17. Bazarian JJ, Cernak I, Noblehaeusslein LJ, Potolicchio S and Temkin N: Long-term neurologic outcomes after traumatic brain injury. J Head Trauma Rehabil 24: 439, 2009.

18. Huang YH, Yang TM, Lin WC, Ho JT, Lee TC, Chen WF, Rau CS and Wang HC: The prognosis of acute blunt cervical spinal cord injury. J Trauma 66: 1441-1445, 2009.

19. Kawano H, Kimura-Kuroda J, Komuta Y, Yoshioka N, Li HP, Kawamura K, Li Y and Raisman G: Role of the lesion scar in the response to damage and repair of the central nervous system. Cell Tissue Res 349: 169-180, 2012.

20. Ziebell JM and Morganti-Kossmann MC: Involvement of pro- and anti-inflammatory cytokines and chemokines in the pathophysiology of traumatic brain injury. Neurotherapeutics 7: 22-30, 2010.

21. Sandhir R, Puri V, Klein RM and Berman NE: Differential expression of cytokines and chemokines during secondary neuron death following brain injury in old and young mice. Neurosci Lett 369: 28-32, 2004.

22. de Haas AH, van Weering HR, de Jong EK, Boddeke HW and Biber KP: Neuronal chemokines: Versatile messengers in central nervous system cell interaction. Mol Neurobiol 36: 137-151, 2007.

23. Rostène W, Dansereau MA, Godefroy D, Van Steenwinckel J, Reaux-Le Goazigo A, Mélik-Parsadaniantz S, Apartis E, Hunot S, Beaudet N and Sarret P: Neurochemokines: A menage a trois providing new insights on the functions of chemokines in the central nervous system. J Neurochem 118: 680-694, 2011.

24. Estes ML and Kimberley MAA: Alterations in immune cells and mediators in the brain: It's not always neuroinflammation! Brain Pathol 24: 623-630, 2014.

25. Morganti-Kossmann MC, Satgunaseelan L, Bye N and Kossmann T: Modulation of immune response by head injury. Injury 38: 1392-1400, 2007.

26. Bowes AL and Yip PK: Modulating inflammatory cell responses to spinal cord injury: All in good time. J Neurotrauma 31: 1753-1766, 2014.

27. Huhtinen A, Hongisto V, Laiho A, Löyttyniemi E, Pijnenburg D and Scheinin M: Gene expression profiles and signaling mechanisms in $\alpha_{2 B}$-adrenoceptor-evoked proliferation of vascular smooth muscle cells. Bmc Syst Biol 11: 65, 2017.

28. Paolicelli RC, Bisht K and Tremblay MË: Fractalkine regulation of microglial physiology and consequences on the brain and behavior. Front Cell Neurosci 8: 129, 2014.

29. Roseti C, Fucile S, Lauro C, Martinello K, Bertollini C, Esposito V, Mascia A, Catalano M, Aronica E, Limatola C and Palma E: Fractalkine/CX3CL1 modulates GABA, currents in human temporal lobe epilepsy. Epilepsia 54: 1834-1844, 2013.

30. Lee HW, Lee K, Kim DG, Yang H and Nam DH: Facilitating tailored therapeutic strategies for glioblastoma through an orthotopic patient-derived xenograft platform. Histol Histopathol 31: 269-283, 2016.

31. Marchesi F, Locatelli M, Solinas G, Erreni M, Allavena P and Mantovani A: Role of CX3CR1/CX3CL1 axis in primary and secondary involvement of the nervous system by cancer. J Neuroimmunol 224: 39-44, 2010.

32. Poniatowski ŁA, Wojdasiewicz P, Krawczyk M, Szukiewicz D, Gasik R, Kubaszewski Ł and Kurkowska-Jastrzębska I: Analysis of the role of CX3CL1 (Fractalkine) and its receptor CX3CR1 in traumatic brain and spinal cord injury: Insight into recent advances in actions of neurochemokine agents. Mol Neurobiol 54: 2167-2188, 2017.

33. de Pablos RM, Herrera AJ, Espinosa-Oliva AM, Sarmiento M, Muñoz MF, Machado A and Venero JL: Chronic stress enhances microglia activation and exacerbates death of nigral dopaminergic neurons under conditions of inflammation. J Neuroinflammation 11: 34, 2014.

34. Gwak YS, Kang J, Unabia GC and Hulsebosch CE: Spatial and temporal activation of spinal glial cells: Role of gliopathy in central neuropathic pain following spinal cord injury in rats. Exp Neurol 234: 362-372, 2012.

35. Zhang L, Zhang J and You Z: Switching of the microglial activation phenotype is a possible treatment for depression disorder. Front Cell Neurosci 16: 306, 2018. 
36. Joshi M and Fehlings MG: Development and characterization of a novel, graded model of clip compressive spinal cord injury in the mouse: Part 1. Clip design, behavioral outcomes, and histopathology. J Neurotrauma 19: 175-190, 2002.

37. Liu YZ, Wang C, Wang Q, Lin YZ, Ge YS, Li DM and Mao GS Role of fractalkine/CX3CR1 signaling pathway in the recovery of neurological function after early ischemic stroke in a rat model. Life Sci 184: 87-94, 2017.

38. Fehlings MG, Wilson JR, Harrop JS, Kwon BK, Tetreault LA Arnold PM, Singh JM, Hawryluk G and Dettori JR: Efficacy and safety of methylprednisolone sodium succinate in acute spinal cord injury: A systematic review. Global Spine J 7 (Suppl 3): S116-S137, 2017.

39. Livak KJ and Schmittgen TD: Analysis of relative gene expression data using real-time quantitative PCR and the 2(-Delta Delta C(T)) method. Methods 25: 402-408, 2001.

40. Cederblad L, Rosengren B, Ryberg E and Hermansson NO: AZD8797 is an allosteric non-competitive modulator of the human CX3CR1 receptor. Biochem J 473: 641-649, 2016.

41. Stavros A and Demetrios S: Chemokines and atherosclerosis: Focus on the CX3CL1/CX3CR1 pathway. Acta Pharmacol Sin 34: 1251-1256, 2013.

42. Berkhemer OA, Fransen PS, Beumer D, van den Berg LA Lingsma HF, Yoo AJ, Schonewille WJ, Vos JA, Nederkoorn PJ, Wermer MJ, et al: A randomizedtrial ofintraarterial treatment for acute ischemicstroke. N Engl J Med 372: 11-20, 2015.

43. Campbell BC, Mitchell PJ, Kleinig TJ, Dewey HM, Leonid C, Nawaf Y, Bernard Y, Dowling RJ, Parsons MW, Oxley TJ, et al: Endovascular therapy for ischemic stroke with perfusion-imaging selection. N Engl J Med 372: 1009-1018, 2015.

44. Goyal M, Demchuk AM, Menon BK, Eesa M, Rempel JL Thornton J, Roy D, Jovin TG, Willinsky RA, Sapkota BL, et al: Randomized assessment of rapid endovascular treatment of ischemic stroke. N Engl J Med 372: 1019-1030, 2015.

45. Sofroniew MV: Molecular dissection of reactive astrogliosis and glial scar formation. Trends Neurosci 32: 638-647, 2009.

46. Hara M, Kobayakawa K, Ohkawa Y, Kumamaru H, Yokota K, Saito T, Kijima K, Yoshizaki S, Harimaya K, Nakashima Y and Okada S: Interaction of reactive astrocytes with type I collagen induces astrocytic scar formation through the integrin-N-cadherin pathway after spinal cord injury. Nat Med 23: 818-828, 2017.

47. Chen Y, Won SJ, Xu Y and Swanson RA: Targeting microglial activation in stroke therapy: Pharmacological tools and gender effects. Curr Med Chem 21: 2146-2155, 2014.
48. Stoll G, Jander S and Schroeter M: Inflammation and glial responses in ischemic brain lesions. Prog Neurobiol 56: 149-171, 1998.

49. Sungho L, Varvel NH, Konerth ME, Guixiang X, Cardona AE, Ransohoff RM and Lamb BT: CX3CR1 deficiency alters microglial activation and reduces beta-amyloid deposition in two Alzheimer's disease mouse models. Am J Pathol 177: 2549-2562, 2010.

50. Zhu W, Acosta C, MacNeil B, Cortes C, Intrater H, Gong Y and Namaka M: Elevated expression of fractalkine (CX3CL1) and fractalkine receptor (CX3CR1) in the dorsal root ganglia and spinal cord in experimental autoimmune encephalomyelitis: Implications in multiple sclerosis-induced neuropathic pain. Biomed Res Int 2013: 480702, 2013.

51. Limatola $\mathrm{C}$ and Ransohoff $\mathrm{RM}$ : Modulating neurotoxicity through CX3CL1/CX3CR1 signaling. Front Cell Neurosci 8: 229, 2014.

52. Bracken MB, Shepard MJ, Holford TR, Leo-Summers L, Aldrich EF, Fazl M, Fehlings M, Herr DL, Hitchon PW, Marshall LF, et al: Administration of methylprednisolone for 24 or $48 \mathrm{~h}$ or tirilazad mesylate for $48 \mathrm{~h}$ in the treatment of acute spinal cord injury. Results of the Third National Acute Spinal Cord Injury Randomized Controlled Trial. National Acute Spinal Cord Injury Study. JAMA 277:1597-1604, 1997.

53. Bracken MB, Shepard MJ, Holford TR, Leo-Summers L, Aldrich EF, Fazl M, Fehlings MG, Herr DL, Hitchon PW, Marshall LF, et al: Methylpredniso-lone or tirilazad mesylate administration after acute spinal cordinjury: 1-year follow up. Results of the third National Acute Spinal Cord Injury randomized controlled trial. J Neurosurg 89: 699-706, 1998.

54. Biber $\mathrm{K}$, Owens $\mathrm{T}$ and Boddeke $\mathrm{E}$ : What is microglia neurotoxicity (Not)? Glia 62: 841-854, 2014.

55. Kasama T, Wakabayashi K, Sato M, Takahashi R and Isozaki T: Relevance of the CX3CL1/fractalkine-CX3CR1 pathway in vasculitis and vasculopathy. Transl Res 155: 20-26, 2010.

This work is licensed under a Creative Commons Attribution-NonCommercial-NoDerivatives 4.0 International (CC BY-NC-ND 4.0) License. 